\title{
EL PAISAJE COMO PATRIMONIO EDIFICADO: UNA REFLEXIÓN HACIA EL PLANEAMIENTO URBANO ${ }^{1}$
}

\author{
LANDSCAPE AS BUILT HERITAGE, A CONSIDERATION TOWARDS URBAN PLANNING
}

\author{
VÍCTOR PEÑA GUILLÉN2 \\ Fecha de recepción: II de julio de 2014. \\ Fecha de aprobación: 25 de agasta de 2014.
}

\section{RESUMEN}

El concepto "patrimonio cultural" se ha convertido en el paradigma de la conservación de aquellos componentes del paisaje urbano que son relevantes por su valor cultural e histórico. Desde inicios de la edad moderna, esta idea ha sufrido una evolución conceptual como término, y ha incorporado gradualmente, primero, los objetos y monumentos, luego las ciudades y paisajes hasta, finalmente, incorporar a las comunidades humanas (Sonkoly, 2010). El paisaje es una idea que permite la comprensión y el estudio del patrimonio edificado, pues su significado abarca la dinámica complejidad de los sistemas urbanos. Según Antrop (2008), el paisaje también ha venido ampliando su significado hasta llegar a ser "un campo de acción de los procesos en el tiempo y el espacio". El presente documento intenta explicar el concepto de patrimonio cultural edificado desde la perspectiva teórica del paisaje, considerando su utilidad en la actividad del planeamiento del desarrollo urbano. En esta tarea, se utiliza el enfoque denominado Paisaje Urbano Histórico (Unesco, 2011), el cual ayuda a demarcar una estructura conceptual que se considera útil en el planeamiento y en la gestión de la conservación del patrimonio edificado en la escala de paisaje.

\section{PALABRAS CLAVE}

Paisaje urbano histórico, paisaje, planeamiento urbano.

\section{ABSTRACT}

The concept of Cultural Heritage has become a conservation paradigm for those urban landscape components of outstanding cultural and historical value. Since the beginnings of modern era, its definition has experienced a conceptual evolution by incorporating progressively first objects and monuments, then cities and landscapes, and finally human communities (Sonkoly, 2010). Landscape is a term that allows study and understanding of built heritage, because now its meaning integrates the dynamic and complex urban systems. Antrop (2008) stated that landscape has been broadening its significance to become a "field of action for temporal and spatial processes". Current document integrates built cultural heritage concept from a landscape theoretical approach, and towards its application onto an urban planning scheme. Another approach called Historic Urban Landscape (Unesco, 2011) helps providing an conceptual framework for this task, which is hypothesized as useful for built heritage planning and management at a landscape scale.

\section{KEYWORDS}

Historic Urban Landscape, Landscape, Urban Planning.

\footnotetext{
1. El documento presentado hace referencia a la dimensión patrimonial urbana, que forma parte de la investigación acerca de la operación de los sistemas sociales y ecológicos urbanos que el autor viene desarrollando como profesor asociado en el Departamento Académico de Ordenamiento Territorial y Desarrollo Sostenible, de la Universidad Nacional Agraria La Molina. Asimismo, la temática viene inspirada y fundamentada en los estudios de maestría en Arquitectura del Paisaje, en la Universidad Agraria de Tokio, y en los cursos del programa de maestría en Renovación Urbana de la Universidad Nacional de Ingeniería en Lima.
}

2. Ingeniero agrícola, especialista en arquitectura del paisaje, sistemas de información geográfica y planeamiento. Es egresado de la UNALM. Cursó la Maestría de Renovación Urbana de la UNI y obtuvo el grado de Máster en Arquitectura del Paisaje en la Universidad de Agricultura de Tokio. Es profesor asociado en la UNALM. Realiza trabajos de aplicación práctica referidos a la identificación, descripción y evaluación del paisaje; sus mayores áreas de trabajo son las zonas costeras y las áreas de operaciones mineras en la sierra peruana. (victorpg@lamolina.edu.pe). 


\section{Introducción}

En los últimos 50 años, el crecimiento de la población mundial y peruana y su agrupamiento en ciudades ha provocado problemas relacionados tanto a la degradación en las condiciones de habitabilidad de la población como al crecimiento desordenado de las ciudades. Este fenómeno de la urbanización, en la escala local, viene poniendo en riesgo la integridad del carácter del paisaje en los núcleos de patrimonio urbano, los cuales sostienen la identidad presente y futura de las ciudades, sobre todo de aquellas que son consideradas de importancia patrimonial.

El concepto "patrimonio cultural" se ha convertido en el paradigma de la conservación de aquellos componentes del paisaje urbano que son relevantes por su importancia cultural e histórica. El patrimonio urbano constituye la más numerosa y diversa selección de patrimonio cultural dentro de la Lista de Patrimonio Mundial de la Unesco. Según Krogius (2006 citado por Trott 2008), del total de 830 sitios naturales y culturales inscritos hasta ese año, 411 lugares pertenecían a ambientes urbanos.

Durante los siglos XIX y XX, el desarrollo urbano en las ciudades industriales da motivo a la aparición de iniciativas de renovación en la estructura edificada de las ciudades; paralelamente, durante la misma época, aparecen y se establecen los principios ideológicos del modernismo, los cuales abogaban por la regeneración y remoción de las viejas estructuras urbanas, y daban validez a las acciones que ponían en peligro la integridad de los componentes históricos y patrimoniales de las ciudades. Estos eventos motivaron la aparición y evolución de la corriente de Conservación Histórica Urbana; con el tiempo, esta corriente se ha ido convirtiendo en un área de especialización y en un concepto de uso corriente entre los conocedores y practicantes de la dimensión profesional referida a la conservación del patrimonio cultural urbano. Si bien la puesta en práctica de este enfoque permitió un avance en el desarrollo, tanto de las teorías como de las técnicas en la especialidad, también supuso el aislamiento de la conservación con respecto a la gestión de los procesos urbanos en el sentido más amplio de la gestión de la ciudad. En los últimos años, la validez de esta aproximación ha sido puesta en discusión y revisión, en tanto que el nuevo siglo está requiriendo una integración mayor de las actividades de conservación y preservación de lo que es llamado histórico, dentro del marco más amplio de la gestión de los procesos de desarrollo urbano (Bandarin y Van der Oers, 2011).

La expansión de la noción de patrimonio cultural ha venido orientando la continua redefinición del llamado patrimonio urbano. Esto ocurre sobre todo en los últimos años del siglo XX, luego del término de la Segunda Guerra Mundial y con la aparición de nuevas corrientes sociales y culturales en los años 60, muchas de las cuales se definen dentro del posmodernismo. De este modo y en adelante, los Centros Históricos protegidos ya no serían considerados solamente un agrupamiento de edificaciones y monumentos estéticamente atractivos o históricamente significantes; la evolución en el enfoque de patrimonio cultural los lleva, a partir de ahora, a ser estudiados como "hábitat social". Así, el proceso de planeamiento urbano toma en cuenta también a los receptores de los cambios (Sonkoly 2010), que pueden ser tanto residentes como transeúntes. Los llamados hábitats sociales deben ser preservados en armonía con su entorno natural, en su sentido amplio de espacio físico y dimensión perceptiva, y a través de la movilización de sus comunidades. Cabe remarcar que esta movilización de los actores involucrados en los procesos de gestión y planeamiento no solo se limita a la información o consulta, sino que se prefiere su participación activa en un esquema que ha devenido en el siglo XXI en la propuesta de planeamiento transdisciplinario.

Los desafíos para la conservación del patrimonio cultural urbano, durante el presente siglo, están muy relacionados con el creciente proceso de urbanización que está sufriendo el mundo; a partir del 2007 más del $50 \%$ de la población mundial está viviendo en espacios urbanos. En medio de este proceso de urbanización, se remarca la importancia de los asentamientos humanos en general y las ciudades históricas en particular. La Declaración de Estambul (1995), emitida durante la Segunda Conferencia de las Naciones Unidas sobre Asentamientos Humanos, sostiene que estos mantienen "la promesa del desarrollo humano y la protección de los recursos naturales del mundo". En la implementación de estos principios, esta declaración compromete a los estados firmantes a abocarse a "la protección de los lugares santos, así como los lugares de significancia histórica y cultural”.

El presente documento describe y discute los instrumentos y definiciones en los temas de patrimonio cultural, paisaje urbano histórico, y planeamiento 
urbano, a partir de la revisión de la bibliografía académica e institucional seleccionada. Estos conceptos son abordados desde el amplio significado que ha adquirido el concepto de paisaje, el cual se utiliza para sintetizar y esquematizar el significado del patrimonio edificado de un modo funcional, y orientado al planeamiento y la gestión del espacio urbano en su dimensión más integral y holística. De este modo, este documento aborda los tópicos descritos siguiendo la siguiente secuencia narrativa: primero, se considera una descripción de la evolución paralela de los conceptos de patrimonio cultural urbano y de paisaje desde los inicios de la Edad Contemporánea hasta el presente; en segundo lugar, se estudia y discute estos dos conceptos tomando en cuenta la complementariedad de ambos; finalmente, el estudio describe la mencionada integración conceptual, entre el paisaje y el patrimonio edificado, para las condiciones de elaboración y delineación de políticas y planes referidos al desarrollo urbano.

\section{Historia de las ideas de patrimonio urbano y paisaje a partir de la edad moderna}

Los últimos 300 años de la historia humana están caracterizados por un incremento demográfico sin precedentes, en particular de la población urbana, lo cual unido a las innovaciones tecnológicas ha provocado un crecimiento de la velocidad, magnitud e impacto de las actividades humanas nunca antes visto sobre la superficie y recursos del planeta (Antrop, 2008).

El concepto de patrimonio, en su concepción moderna, aparece durante la Revolución Francesa, que fue el periodo en el que juntamente con la destrucción de los monumentos y edificaciones del antiguo régimen se proveyeron las primeras medidas institucionales referidas a la conservación del patrimonio en el sentido que se le conoce actualmente. Las ordenanzas, emitidas en esa época, sugieren medidas de conservación en el entendido que la preservación del patrimonio cultural es importante para los propósitos institucionales orientados a "mantener el liderazgo de Francia en el comercio y la industria" (Jokilehto, 1986).

El modernismo rechazó la conservación y definió la ciudad histórica como un área separada de la ruta del desarrollo urbano, dejando de lado la amplia dimensión conceptual propia de la práctica de este último. El periodo posterior a la Segunda Guerra Mundial fue testigo de la extensiva destrucción planificada de muchas áreas históricas urbanas en varias partes del mundo. Esta alteración estuvo asociada con la necesidad de responder a los grandes flujos migratorios del campo a la ciudad, con el incremento en la tenencia de vehículos privados y con la presión generada por la inversión inmobiliaria para usos residenciales y comerciales (Bandarin y Van der Oers, 2011).

Durante la ocurrencia de estos cambios, la conciencia acerca de la preservación estuvo presente; la preocupación por la conservación de los monumentos históricos tiene reflejo, por ejemplo en las conclusiones de la Conferencia de Atenas (1931), que dio lugar a la llamada Carta de Atenas para la restauración de edificios y monumentos históricos. Asimismo, es notoria la importancia, provista a nivel internacional a ese tema, en las disposiciones emitidas por la Convención para la Protección de la Propiedad Cultural en el evento de un Conflicto Armado, suscrito en La Haya en 1954. Si bien el enfoque de ambos eventos es sobre los objetos o monumentos individuales, la percepción creciente se orienta a su integración dentro del contexto geográfico general donde estos se ubican.

Respecto de la Carta de Atenas, cabría resaltar su importancia como antecedente conceptual y metodológico en la evolución de la idea de patrimonio cultural urbano. En el aspecto conceptual, las conclusiones elaboradas al final de la conferencia introducen importantes principios como la idea de un patrimonio común de los habitantes de la Tierra; el principio de integración de nuevos materiales en la restauración de monumentos; y, algo importante en la presente discusión, que es la importancia del contexto espacial en los monumentos (The Getty Conservation Institute 2014). Metodológicamente, también se sugieren medidas como por ejemplo la práctica de volver a enterrar los sitios arqueológicos que no vayan a ser restaurados. Finalmente. se inicia la discusión acerca de la necesidad de crear una asociación de arquitectos y especialistas de edificios históricos, independientes de la Asociación Internacional de Museólogos, que a su vez era una asociación de especialistas en conservación de monumentos históricos, la cual fue la organizadora del evento de Atenas. Las inquietudes descritas líneas arriba, originan las reuniones de París (1957) y de Venecia (1964), las cuales fueron respectivamente el Primer y Segundo Congreso Internacional de Arquitectos y Especialistas de Edificios Históricos (Icomos, 2004). 
En el primer congreso, se recomienda por ejemplo el establecimiento de autoridades o instituciones para la protección de edificios históricos. En una de las conclusiones se sugiere que los arquitectos y los planificadores urbanos deben cooperar para la integración de los edificios históricos dentro del planeamiento de la ciudad. El segundo congreso daría lugar a la famosa Carta de Venecia suscrita más de 30 años después de la Carta de Atenas (1964) la cual extiende el concepto de monumento histórico al establecer que este "abarca no solo la obra arquitectónica singular, sino que también el contexto urbano y rural donde se encuentra la evidencia de una civilización, un desarrollo particular o un evento histórico". Esto se aplica no solo a las obras de arte; también, a los objetos modestos del pasado, los cuales han adquirido un significado cultural con el paso del tiempo (Bandarin y Van der Oers, 2011).

El reconocimiento de los cambio ocurridos en los paradigmas sociales y culturales, durante la década de los sesenta, parece también haber influenciado en la maduración del significado de patrimonio cultural; de este modo, no puede ser casualidad que estos cambios dieran forma a las ideas propuestas y aprobadas en la Convención sobre Patrimonio Mundial del año 1972. Entre los cambios ideológicos y culturales, que enmarcan la evolución de la noción de patrimonio cultural, Sonkoli (2010) menciona los siguientes:

- La perspectiva modernista orientada hacia el futuro fue reemplazada o al menos emparejada por el encuadre postmoderno hacia el tiempo presente.

El termino de patrimonio cultural gradualmente se fue incorporando en casi todos los dominios del pasado (primero en los objetos y monumentos, luego en las ciudades, paisajes y comunidades humanas).

- Las grandes teorías que explican el desarrollo social fueron puestas en cuestión.

- Tanto en las humanidades como en las ciencias sociales (que incluyen a la economía y los estudios urbanos) se llevó a cabo un cambio en la estructura de sus propios paradigmas.

- Los cambios mencionados en los campos lingüísticos y culturales se acomodan perfectamente en la multiplicación de identidades.

- La multiplicación de identidades y el empoderamiento de las voces locales dieron lugar a la ampliación del alcance de la democratización y el reconocimiento académico de dichas identidades descubiertas.
La aparición y el reconocimiento de la importancia que podría tener para la Humanidad el proceso acelerado de urbanización, promueve la Primera Conferencia de las Naciones Unidas sobre Asentamientos Humanos - Hábitat I, llevada a cabo en Vancouver en 1976. En ella, se emiten tanto la Declaración de Vancouver como el Plan de Acción de Vancouver. Uno de los principios instaurados en la declaración señala que los asentamientos históricos, monumentos y otros elementos del patrimonio nacional deben ser protegidos frente a cualquier acto de agresión o abuso del poder de turno. En el Plan de Acción, se recomienda la adopción de una política integrada en asentamientos humanos, la cual debe ser consistente con la preservación, restauración y mejora de los ambientes naturales y construidos, que reconozca el rol positivo del ambiente en el desarrollo económico y social de las naciones.

Uno de los dos imperativos establecidos en la Declaración de Estambul sobre Asentamientos Humanos, durante la Segunda Conferencia de las Naciones Unidas sobre Asentamientos Humanos (1995), considera abordar el desafío del desarrollo sostenible de los asentamientos humanos en un mundo urbanizado. En tal sentido, la declaración establece una visión integral para el desarrollo de los asentamientos humanos, dentro de los cuales se encuentran las ciudades históricas; el panorama general incluye y combina los desarrollos social y económico y la protección ambiental. Esta premisa provee el sustento inicial de toda intervención que busque el bienestar del hombre, al ser este el fin último y el centro de la preocupación del desarrollo sostenible. Asimismo la declaración señala explícitamente la necesidad de mantener presentes el significado de las particularidades nacionales y regionales, así como los diferentes antecedentes culturales, religiosos e históricos, lo cual configura la identidad del paisaje.

El Memorándum de Viena (Unesco, 2005) introduce el concepto de "Paisaje Histórico Urbano", el cual se describe a partir de su composición, y la integran elementos de un carácter definido que incluyen a los patrones y usos del suelo, la organización espacial, las relaciones visuales, la topografía y los suelos, la vegetación y también todos los elementos de infraestructura que incluyen objetos de pequeña escala y detalles constructivos. A los componentes del paisaje señalados se pueden agregar elementos particulares de los panoramas urbanos, como los panoramas de los techos, los ejes visuales principales, los lotes y las 
tipologías de edificación, los cuales son parte integral de la identidad del Paisaje Histórico Urbano.

La Recomendación sobre Paisaje Urbano Histórico fue aprobada por la Unesco, en su Conferencia General llevada a cabo en París el año 2011. En este documento, el concepto de Paisaje Urbano Histórico se propone como una aproximación innovadora destinada a preservar el patrimonio y gestionar ciudades incluidas en la Lista de Patrimonio Mundial.

El contexto geográfico al cual se refieren los documentos mencionados líneas arriba representa al espacio urbano como una integralidad en sus dimensiones física y perceptiva; a lo largo de su evolución conceptual el patrimonio cultural ha incorporado gradualmente primero a los objetos y monumentos, luego las ciudades, paisajes hasta finalmente incorporar a las comunidades humanas (Sonkoly, 2010). El patrimonio cultural es definido y caracterizado por su historia, y su significado en el contexto urbano. La lógica sostenida en el presente documento sostiene que el concepto de paisaje, en su noción de carácter total, puede proveer un marco teórico y funcional para entender el nuevo enfoque del Paisaje Urbano Histórico, orientado al planeamiento urbano.

La definición de paisaje enunciada por Humboldt identifica al paisaje como una singular síntesis entre las características naturales y culturales de una región (Antrop 2005); según este eminente geógrafo y naturalista, el paisaje es "el carácter total de una región de la tierra” (Zonneveld 1995, citado por Antrop 2005). Según Kwa (2005), el concepto aparece como una síntesis de los sistemas natural y cultural observados por Humboldt durante sus viajes. De este modo, Humboldt va más allá de la concepción pictórica o simbólica, que proviene de la tradición occidental desde la época del Renacimiento, señalando que los sistemas natural y cultural conforman una unidad holística representada en el concepto de paisaje.

La Convención Europea del Paisaje (Concilio de Europa 2000) define al paisaje como el área percibida por la gente, cuyo carácter es el resultado de la acción e interacción de los factores naturales y humanos. A partir de la definición de la Convención, Antrop (2008) señala que el paisaje tiene las siguientes características:

Es visto como una entidad espacial, teniendo una escala y extensión variables, y propiedades territoriales.
Es el objeto percibido y experimentado por los humanos.

Está compuesto por muchos y muy diferentes elementos y componentes que interactúan entre sí y están estructurados en un arreglo particular.

Tiene una organización y gestión espacial que son influenciadas por los humanos.

El término Paisaje Histórico Urbano muestra también un carácter holístico, pues se define como el área urbana entendida como el resultado de la estratificación histórica de los valores y atributos culturales y naturales; de ese modo, esta comprensión se extiende más allá de la noción de centro histórico para incluir el más amplio contexto y localización urbana (Unesco, 2011).

El enfoque de Paisaje Histórico Urbano se dirige más allá de la preservación del medioambiente físico, pues se enfoca en el medio ambiente humano completo, que incluye todas sus cualidades tangibles e intangibles. Los recursos tangibles o físicos del Paisaje Urbano Histórico abarcan todos los elementos naturales y construidos en la geografía urbana. Entre ellos se encuentran los siguientes:

- Sistemas naturales, geomorfología, hidrología, topografía.

Vegetación de todos los tipos.

- Sistemas de transporte y circulación.

- Cuerpos de agua naturales y construidos.

Edificaciones.

Arte urbano, esculturas, mobiliario y objetos urbanos.

Los recursos intangibles del Paisaje Urbano Histórico son:

- Festivales tradicionales, rituales, música, danza;

- Adoración espiritual, peregrinación celebración religiosa;

- Lugares icónicos compartidos, aquellos símbolos que encarnan la identidad urbana;

Culinaria regional y local.

Por lo señalado en este capítulo, la aproximación del Paisaje Urbano Histórico es holística y por tanto, comprende un sistema donde los recursos tangibles e intangibles están interrelacionados y se influencian entre sí. Asimismo, se ha observado una evolución progresiva en el grado de amplitud del área de estudio y su significado; inicialmente, se consideraba al espacio físico del área histórica como el espacio 
delimitado, donde se agrupan un conjunto de elementos arquitectónicos con características históricas similares; luego, se adopta el concepto de Panorama Urbano que incluye además la percepción, aunque se estudia todavía aislado de la permanente dinámica de lo urbano no solo en la superficie que ocupa el patrimonio edificado, sino más allá todavía, en el contexto de la región urbana. Finalmente, aparece el enfoque que asume una dimensión holística donde el patrimonio urbano existente se relaciona con la ciudad no solo en sus dimensiones históricas, sino también sociales y económicas. Es el soporte patrimonial de la identidad de toda la ciudad y se acepta que con el paso del tiempo, las estructuras y procesos físicos irán cambiando, así como también la percepción que se modifica conforme lo hace la dimensión física.

\section{Los conceptos de patrimonio y paisaje frente al panorama de cambio}

Los conceptos de patrimonio cultural y la actividad del planeamiento del desarrollo urbano hacen frente a la realidad del cambio permanente en el paisaje urbano utilizando como referencia justamente al concepto de paisaje.

Las ciudades son organismos dinámicos; no existe alguna ciudad "histórica" en el mundo que haya retenido su carácter "original"; este concepto de ciudad se asemeja a un blanco móvil destinado a cambiar con la misma sociedad. Para preservar el Paisaje Histórico Urbano, se requiere construir alianzas estratégicas y dinámicas entre los diferentes actores presentes en la escena urbana, sobre todo entre los residentes, los transeúntes (turistas), las autoridades públicas que gestionan la ciudad, así como con los desarrolladores inmobiliarios y empresarios que operan e invierten en la ciudad (Unesco, 2013).

Si bien los paisajes han cambiando a lo largo de la historia de la civilización humana, desde el punto de vista de los actores involucrados, en el pasado, estos cambios fueron el reflejo de iniciativas de un número pequeño de grandes propietarios (Muir 2003, citado por Antrop, 2008). Sin embargo, a partir de las iniciativas de democratización, llevadas a cabo durante el siglo XX, y que trajeron como resultado el incremento en la cantidad de pequeños propietarios, se produjeron muchos cambios en los usos del suelo de pequeña escala y no fueron concordados entre sí. Este incremento sustancial en el número de actores (sobretodo privados) con competencia territorial ge- nera un efecto acumulativo que causa el cambio, no siempre planificado y más que todo caótico (Antrop 2008).

Las ciudades históricas vienen desarrollándose en un contexto de presiones externas entre las cuales se reconocen: la urbanización, el cambio climático, la explotación mercantil y el turismo de masas. Estos factores influencian y mantienen la permanencia del cambio, motivando la construcción de nuevos escenarios donde el Paisaje Urbano Histórico es modelado de forma aleatoria, quizá como producto de una identidad débil, y con planes sin modos y actividades efectivas de aplicación o implementación que dejan en condición vulnerable al Paisaje Urbano Histórico. Esencialmente, la globalización es la fuerza que dirige el cambio a escala local; en tal sentido, los motores, valores que orientan el cambio, provienen desde todos lugares.

La Recomendación sobre Paisaje Histórico Urbano (Unesco 2011) reconoce al proceso de urbanización como el causante de la fragmentación social y espacial de la ciudad, así como de un drástico deterioro en la calidad del ambiente urbano. Entre sus efectos evidentes se mencionan los siguientes: excesiva densidad urbana, edificios monótonos y estandarizados, pérdida de espacios públicos, inadecuada infraestructura, pobreza, aislamiento social y un creciente riesgo de desastres relacionados al cambio climático.

De acuerdo con O'Donnell, las principales presiones sobre el patrimonio urbano durante el siglo XXI serán consecuencia del crecimiento de la población urbana en el planeta por sobre el 50 \% de la población total, lo cual da como resultado un panorama demográfico caracterizado por un crecimiento incontrolado. Se estima que para el año 2030 la población urbana corresponderá al $75 \%$ del total de la población del planeta.

Según Antrop (2004), los paisajes culturales son el resultado de la reorganización consecutiva del territorio, de modo que mejor se adapte, tanto su uso como su estructura espacial, a las demandas sociales cambiantes. Los cambios en el paisaje son vistos como una amenaza, una evolución negativa, debido a que son caracterizados por la pérdida de la diversidad, coherencia e identidad de los paisajes existentes. Antrop (2008, citando a Fairclough, 2003) menciona que el cambio no se refiere a aquello que le sucede a los paisajes, no es la consecuencia, es más bien la causa y por tanto, es el cambio el que construye a los 
paisajes. La lógica recomendación que se desprende desde esta última idea sugiere que los paisajes deben asumir el cambio como un proceso natural, aceptándolo y adaptándose a ellos.

Las presentes condiciones de cambio acelerado en la sociedad y el medio ambiente resultan en la creación de paisajes completamente nuevos y asimismo, en el rápido deterioro de aquellos tradicionales, lo cual es considerado como una amenaza y pérdida de la calidad y los valores. En todo caso, no existen razones para considerar que los paisajes tradicionales son mejores que los paisajes contemporáneos (Antrop, 2008), a menos que se refieran aquellos de calidad remarcable y que han sido considerados, por ejemplo, dentro de la reconocida Lista de Patrimonio Mundial de la Unesco.

La presión de las actividades humanas sobre el Paisaje Urbano Histórico, sobretodo en ciudades donde predomina la economía de libre mercado, se debe también a la existencia de una competencia internacional entre ciudades, en lo referido tanto al crecimiento económico, como al ingreso por la actividad turística.

Durante la primera mitad de la década de los sesenta del siglo pasado, la población peruana se convirtió en mayoritariamente urbana (United Nations Population Division, 2011). El mencionado proceso de urbanización ha venido modificando a gran velocidad el aspecto físico de las ciudades peruanas. Los cambios se han notado tanto en la dimensión natural como en la construida. En muchas ciudades, se ha venido cambiando el uso de los valiosos terrenos agrícolas hacia un uso principalmente residencial; asimismo, los centros urbanos están siendo renovados o afectados por inversiones inmobiliarias, de carácter comercial o institucional, que ponen en riesgo las características físicas y también, los valores y percepciones de la comunidad que los habita y visita. A modo de ejemplo práctico de la ocurrencia de algunos eventos, que resaltan los aspectos comunes entre patrimonio y paisaje en la realidad peruana, se puede mencionar el conflicto entre autoridades acerca de la licencia otorgada a una nueva edificación en la ciudad del Cusco; el conflicto aparece debido a que el edificio proyectado bloquearía el valorado panorama visual desde un conocido mirador hacia el centro histórico de la ciudad, que como se ha dicho tiene un régimen especial en su condición de Patrimonio Mundial (La República, 2014).
En el Perú, tres ciudades han sido incluidas dentro de la Lista de Patrimonio Mundial de la Unesco. El sector involucrado y las fechas de la declaratoria son las siguientes: Centro Histórico de Lima (1991), Centro Histórico de Arequipa (2000) y Ciudad del Cusco (1983). La condición futura en el Paisaje Urbano Histórico de estas tres ciudades viene siendo abordada a partir de instrumentos de gestión, referidos principalmente a los planes maestros. En estos documentos, se establecen las políticas de conservación y restauración, se delinea el Plan General de Usos del Suelo y se proponen usos específicos e intervenciones en las zonas pertenecientes tanto en el sector declarado de interés (Centro Histórico), como en las zonas adyacentes al perímetro de esta, también llamada Zona de Transición. Estos planes reconocen implícitamente la dimensión integral del paisaje como espacio físico relacionado con el observador a través de los usos y percepciones, aunque este último factor no es desarrollado en detalle.

El paisaje no está acotado por bordes, sino que trasciende las delimitaciones político administrativas, pues abarca las vistas en toda su extensión, a menos que sean bloqueadas por algún objetivo físico (edificios, arboles, cerros, etc.). Desde el punto de vista conceptual y académico, esta falta de límites en el alcance de su extensión espacial, permite al amplio abanico de actores sociales identificarse asimismo con su territorio. El concepto de paisaje incluye la percepción del objeto físico como una dimensión fundamental para su observación y estudio. La idea de calidad visual se enmarca dentro de esta noción de percepción, a pesar de que la evaluación de la calidad visual es subjetiva. El método para hacer operativa y sistemática su calificación, intenta describir de forma objetiva la calidad visual a partir de las características físicas de sus componentes.

\section{Complementariedad en los conceptos de paisa- je y patrimonio cultural}

La Convención Europea del Paisaje (2000) define la protección del paisaje como el conjunto de las acciones necesarias para mantener los elementos característicos o significantes de un paisaje. Uno de los principios generales de esta indica reconocer al paisaje, dentro de los instrumentos legales de un estado, como un componente esencial del contexto que rodea a la población, una expresión de la diversidad de su compartido patrimonio natural y cultural, y la base de su identidad. Antrop (2008) refiere que el 
paisaje es considerado como el patrimonio común de una comunidad social, lo cual además lo convierte en difícil de gestionar y proteger; señala asimismo que las acciones en el planeamiento del paisaje deben pasar a través del planeamiento de los usos del suelo. Finalmente, el mencionado autor (citando a Palang, 2004; Tress, 2003; Antrop, 2003; Fry, 2003; Fairclough y Rippon, 2002; Haines-Young ,2000) señala que el paisaje ha llegado a ser un concepto integrador para el planeamiento espacial, el desarrollo sostenible, la gestión ambiental y la protección del patrimonio.

Adicionalmente a la definición de paisaje, la Convención ha formulado las siguientes definiciones:

- Política de paisaje que es la expresión proveniente de las autoridades públicas competentes acerca de los principios generales, estrategias y lineamientos que permiten tomar medidas específicas orientadas a la protección, gestión y planeamiento de los paisajes.

- Objetivos de calidad del paisaje, los cuales se refieren a las aspiraciones del público en relación a los componentes específicos del paisaje y sus alrededores deben ser formulados por las autoridades públicas competentes.

- Protección del paisaje. Esta definición se refiere a todas las acciones dirigidas a conservar y mantener los elementos característicos y significativos de un paisaje, los cuales están justificados a partir de su valor patrimonial y se relacionan con una configuración natural o actividad humana específica.

- Gestión del paisaje que cubre todas las acciones que aseguran el mantenimiento regular de un paisaje y orientan y armonizan los cambios, considerando los procesos sociales, económicos y ambientales a partir de la perspectiva del desarrollo sostenible.

- Planeamiento del paisaje. Es el grupo de vigorosas acciones dirigidas hacia el futuro para reforzar, restaurar y crear paisajes.

En los inicios de la Edad Moderna aparecen los dos conceptos en su forma actual. El paisaje es definido por Von Humboldt (Zonneveld, 1995) como el carácter total de una región sobre la tierra y el patrimonio cultural como los elementos materiales e inmateriales de valor superlativo, que son el legado de generaciones previas.

El término paisaje puede ser utilizado en sus acepciones abstractas y concretas; de forma abstracta, el paisaje no tiene bordes o límites y se refiere a conceptos como panorama, sistema o estructura; en su significado concreto, diversos paisajes se diferencian uno de otro a través de tipos que ocupan una determinada y delimitada superficie. El carácter holístico del paisaje se puede definir de tal modo que este solo obtiene su significado o valor de acuerdo a su contexto o a los elementos que lo rodean. Esta característica del paisaje provoca las siguientes consecuencias (Antrop 2000):

El valor de un elemento no es absoluto: el mismo elemento del paisaje puede tener un valor elevado o bajo de acuerdo a su localización geográfica, lo cual influye en su accesibilidad.

- Al cambiar un elemento, se modifica el todo.

- Al cambiar el contexto, cambia la calidad del elemento incluido en él.

El aspecto perceptivo del paisaje también es importante y determina la forma en la que se considera el ambiente observado, de un modo que es tanto holístico como relativo. La forma como el ser humano percibe el paisaje puede ser descrito como un todo holístico, el cual es más que la suma de sus partes (Antrop, 2000). El carácter perceptivo indica que el paisaje es esencialmente una realidad ante los ojos del espectador y solo puede ser considerado en su completa amplitud cuando se considera también a este (Olwig, 2002, 2004; citado por Antrop, 2005). La percepción como un proceso de aprendizaje complejo analiza la observación, inmediata e interactivamente, enlazando los resultados con el conocimiento y la experiencia del observador (Antrop, 2000).

Entre todas las ciudades incluidas en la Lista de Patrimonio Mundial de la Unesco, solo unas cuantas consideran específicamente la protección de vistas o panoramas urbanos; entre estas ciudades están por ejemplo Budapest y París; la mayoría de las áreas urbanas protegidas se refieren a monumentos o espacios construidos de interés histórico. Se estima que el concepto de paisaje urbano histórico es la solución al problema de la integridad visual del paisaje urbano y viene a ser un nuevo paradigma en el planeamiento urbano (Sonkoly, 2010).

El enfoque provisto por el concepto Paisaje Urbano Histórico tiene como objetivo preservar la calidad del ambiente humano y reforzar la productividad de los espacios urbanos. Este concepto integra las metas de la conservación del patrimonio urbano con las del desarrollo social y económico. El enfoque está fundamentado en las relaciones armoniosas y sosteni- 
bles entre los ambientes natural y construido (Unesco, 2011).

Según Nishimura (2011), este enfoque está orientado hacia la salvaguarda del área de interés, utilizando principalmente un sistema de planeamiento más que una nueva categoría de patrimonio cultural; asimismo, en este enfoque se incluyen una amplia variedad de las dimensiones así como la percepción del Paisaje Urbano Histórico. Este autor sostiene que el estudio y la gestión de estas áreas deben ser abordados siguiendo una secuencia que primero debe considerar el contexto general, y a partir de este dirigirse hacia los elementos particulares o de detalle. Por ejemplo, se sugiere que los exámenes de autenticidad material, para postular a la lista de patrimonio cultural, se deben realizar una vez que se haya revisado la integridad del sitio y su contexto geográfico total o paisaje.

La autenticidad "considerada de esta manera y afirmada en la Carta de Venecia, aparece como el factor esencial en el momento de la calificación de valores culturales de una ciudad que es o pretende ser parte de la Lista de Patrimonio Mundial. La comprensión de la autenticidad juega un papel fundamental en todos los estudios científicos del patrimonio cultural, en la planificación de la conservación y la restauración, así como en los procedimientos de registro de la Convención de Patrimonio Mundial y otros inventarios de patrimonio cultural". (Documento de Nara, 1994).

El Documento de Nara también sugiere que "el patrimonio cultural sea considerado y juzgado dentro del contexto cultural al cual pertenece" el cual puede ser muy diverso. En todo caso, como el paisaje urbano normalmente es dinámico y heterogéneo, la diversidad ha empezado a verse como un mérito.

El debate acerca del futuro de la conservación urbana, enmarcado en torno del concepto del Paisaje Urbano Histórico, muestra el interés existente en la comunidad científica y profesional por reevaluar las viejas prácticas adoptadas en los últimos 50 años. En este ambiente de cambio, la necesidad de esta renovación proviene de diferentes factores como: el reconocimiento del modo en que la diversidad cultural afecta los valores y enfoques hacia la conservación; la evidente relación entre los factores naturales y culturales y su influencia sobre la conservación del patrimonio edificado; los nuevos desafíos provenientes de los rápidos cambios económicos y sociales; el creciente rol y estatus de las ciudades históricas como centro de las actividades artísticas y de los negocios creativos y, finalmente, la necesidad de asegurar un futuro sostenible para la conservación del patrimonio (Bandarin y van Oers, 2011).

\section{Planeamiento sostenible transdisciplinario y conservación del patrimonio urbano}

Desde la adopción de la Convención sobre Patrimonio Mundial (1972), los países miembros de la Unesco se comprometieron a asegurar en lo posible la apropiada identificación, protección, conservación y presentación del patrimonio cultural del mundo. Al mismo tiempo, la comunidad internacional ha adoptado el concepto de desarrollo sostenible, en el entendido que la protección y conservación del patrimonio natural y cultural se consideran una significativa contribución al desarrollo sostenible (Unesco, 2012). La conservación del patrimonio urbano es una práctica de planeamiento en muchos países (Bandarin y van Oers, 2011). La conservación en la ciudad se enmarca en el deseo universal por lograr ambientes urbanos saludables y vitales; es un objetivo que viene remarcándose durante el presente siglo y que todavía no es alcanzado.

Según la Convención Europea del Paisaje (2000), el planeamiento es la poderosa acción anticipatoria dirigida a reforzar, restaurar o crear paisajes. El planeamiento del paisaje aborda paisaje heterogéneo y dinámico; el comportamiento de estos paisajes puede describirse y entenderse de modo semejante a un blanco móvil, en el que el movimiento o cambio siempre estará presente.

Bandarin y Van der Oers (2011) sugieren estudiar tres tópicos de importancia para la conservación urbana en el contexto de cambio actual:

La necesidad de abordar los retos de la conservación urbana frente a las fuerzas de cambio asociados a la globalización, como el turismo de masas

La necesidad de integrar la conservación con el desarrollo y planeamiento urbano dentro de un proceso unitario y no aislado de los planes de desarrollo

La necesidad de revisar los paradigmas clásicos de conservación, con el fin de reconocer la diversidad cultural y la naturaleza dinámica del patrimonio urbano.

Se debe aclarar que a pesar de estar hablando de identidad de la región geográfica, hay que remarcar el rol del centro de la ciudad como guardián de esa identidad. El centro no puede ser descrito, a través 
de sus únicas funciones, por simple oposición con otras partes de la ciudad, o por sus conocidos valores estéticos. El planeamiento urbano ha experimentado su giro cultural al ser reemplazadas gradualmente las ideologías por las identidades. La dimensión histórica y cultural es central en el carácter del paisaje y su comprensión es esencial en la gestión del paisaje (Fairclough, 2004).

En Inglaterra, el Programa para la Caracterización Histórica del Paisaje ha ampliado el alcance de lo que debe ser el patrimonio en términos de forma y función, de composición y de antigüedad. Este programa utiliza un concepto unificador para complementar la tradicional protección de monumentos. La primera lección de dicho programa es aquella que sostiene que la naturaleza y la cultura no están separadas (rechazando la idea que un área determinada es un paisaje natural, mientras que la otra es un paisaje cultural); de esta manera, todos los paisajes son el resultado de la interacción recíproca y a largo plazo entre la naturaleza y la cultura. Este método es más apropiado para gestionar el cambio en el paisaje antes que para su protección. Normalmente, la protección utiliza técnicas de inventario, en cambio la caracterización histórica del paisaje captura un panorama general en el que la información así obtenida sirve para interpretar el carácter del paisaje (Fairclough, 2004).

El paisaje actual es el resultado de procesos históricos y actividades humanas; asimismo, es la consecuencia de otro tipo de procesos culturales, que son aquellos que influencian y estructuran el modo en que la gente los percibe; estas percepciones cambian a medida que el tiempo pasa y el mundo cambia. De este modo, los procesos históricos que crean el paisaje, tanto físicamente como a través de la percepción, son centrales para la gestión del paisaje (Fairclough, 2004).

Una visión del paisaje que reconoce un horizonte temporal a los procesos históricos y al cambio ocurrido en el pasado y el que ocurrirá hacia el futuro, y que reconoce que también son dinámicas la percepción y la apreciación del paisaje, tiene efectos en la manera cómo son abordados la protección, gestión y planeamiento del paisaje. Para poner en práctica la gestión del paisaje, tal como es entendida por la Convención Europea del Paisaje, con el paisaje como núcleo de una política social relevante y con significado para todos, se requiere un énfasis en el reconocimiento del cambio. El cambio va desde el primitivo proteccionismo sectorial (que fue una reacción inicial frente a los elevados niveles de amenazas y pérdidas), hacia una gestión del cambio más positiva, flexible y holística, como parte del planeamiento espacial, el cual debe ser considerado más como un modo de reconocer y afrontar el proceso de cambio, en lugar de oponerse a él (Fairclough 2004).

Para abordar el tópico de la implementación de los planes, es conveniente referirse a lo señalado en el Memorándum de Viena (1995). Este documento señala que la gestión de los cambios y desarrollos dinámicos, en los paisajes urbanos históricos pertenecientes a la Lista de Patrimonio Mundial de la Unesco, requiere un preciso conocimiento del territorio, y sus elementos de significancia patrimonial. Estos factores son identificados en un plan a través de métodos científicos de inventario; en este se incluye la referencia normativa pertinente, y finalmente, las herramientas y procedimientos para su implementación. Para el caso específico del Patrimonio Mundial, el plan se formaliza siguiendo las pautas mencionadas en la Guía Operativa para la Implementación de la Convención de Patrimonio Mundial (2012).

El desarrollo e implementación del Plan de Gestión para los paisajes urbanos históricos requiere la participación de un equipo interdisciplinario de expertos y profesionales y también de la oportuna y amplia integración de actividades de consulta pública. Tress et al. (2005) establecen cuatro niveles de inclusión, de la población o comunidad interesada, dentro del esquema de planeamiento transdiciplinario: información, consulta, participación y control. Tal como se observa en la secuencia progresiva de estos niveles el grado de intervención y empoderamiento va incrementándose. El grado de intervención también influye en su inclusión dentro de las etapas del proceso de planeamiento: definición de los objetivos, inventario, evaluación de las condiciones presentes, propuesta de cambio, implementación y monitoreo.

La gestión de la calidad del paisaje histórico urbano se orienta hacia la permanente preservación y mejora de los valores espaciales, funcionales y de diseño. $\mathrm{Al}$ respecto, se pone un énfasis especial en la contextualización de la arquitectura contemporánea dentro del paisaje urbano, señalándose que los estudios de evaluación de impactos culturales y visuales deben acompañar a las propuestas de intervención contemporáneas. Asimismo, los aspectos económicos del 
desarrollo urbano deben alinearse con los objetivos a largo plazo de la preservación del patrimonio.

Los edificios históricos, los espacios públicos y la arquitectura contemporánea contribuyen significativamente a los valores de la ciudad identificando el carácter de ella. La arquitectura contemporánea puede ser una fuerte herramienta en el incremento de la competitividad de las ciudades, pues de este modo atrae residentes, turistas e inversión. La arquitectura histórica y contemporánea constituye un bien económico para las comunidades locales, el cual sirve a los propósitos educacionales, de recreación, turismo y aseguran el valor de mercado de las propiedades.

\section{Conclusiones}

Son motivo de reflexión y discusión en el presente documento el entendimiento del alcance de los instrumentos de políticas y de gestión del patrimonio esbozados por la Unesco, y aceptados por los países suscritos, la pertinencia del concepto de paisaje en su concepción holística y la complementación de los conceptos patrimonio y paisaje para su aplicación a las condiciones, que actualmente prevalecen en las ciudades peruanas incluidas en la Lista de Patrimonio Mundial,

La revisión bibliográfica se orienta hacia aquellos tópicos referidos al patrimonio cultural edificado y a su enlace con el concepto de paisaje, cuyo significado se utiliza para remarcar el carácter integral del enfoque estudiado; asimismo, el estudio busca orientar la revisión hacia su aplicación dentro del marco metodológico del planeamiento, a fin de descubrir una noción fundamentada para la creación de condiciones futuras adecuadas en un contexto en el que el paisaje es dinámico. Se entiende que el enfoque de la Unesco establece políticas y conceptos que pueden ser útiles para la construcción de los instrumentos institucionales de gestión para cada ciudad en particular y a través del planeamiento.

La aparición de la noción moderna de patrimonio en la historia de la civilización está relacionada con el establecimiento de los Estado-Nación actuales, y de la necesidad de establecer sus propias tradiciones e identidades. Durante los siglos XIX y XX, el patrimonio cultural en las ciudades industrializadas fue alterado y degradado con el objetivo de proveer las condiciones de salubridad e infraestructura que requería la población urbana. Los motivos originales de renovación urbana fueron ideológicamente apo- yados por los dictados del Movimiento Moderno, que abogaba por la regeneración y remoción de las viejas estructuras urbanas, dando validez a las acciones que pusieron en peligro la integridad de los componentes históricos y patrimoniales de las ciudades (Bandarin y Van der Oers, 2011).

El paisaje es un fenómeno complejo que puede ser analizado de diversas maneras (Antrop 2000); el Paisaje Urbano Histórico es una nueva aproximación y a la vez herramienta de gestión; en tal sentido, cabe aclarar que no se le considera simplemente una nueva categoría de protección. El Paisaje Urbano Histórico parece ser el término apropiado para describir el contenido de los centros urbanos históricos contemporáneos (Sonkoly, 2010).

Cabe señalar que la aproximación de Paisaje Urbano Histórico se sustenta en los principios, para el desarrollo del ambiente urbano, que fueron establecidos en la Segunda Conferencia de las Naciones Unidas sobre Asentamientos Humanos (1996). La declaración resultante de dicha conferencia hace un llamado para promover la conservación, rehabilitación y el mantenimiento de edificios, monumentos, espacios abiertos, paisajes y patrones de asentamiento de valor histórico, cultural, arquitectónico, natural, religioso y espiritual.

Una de las responsabilidades asignadas a los estados que suscriben la convención y las recomendaciones sobre Paisaje Urbano Histórico (Unesco, 2011) es la de integrar la protección del patrimonio dentro de los programas integrales de planeamiento. En este sentido, el enfoque para la gestión de los paisajes urbanos históricos es holístico; integra los objetivos de la conservación del patrimonio urbano con aquellos del desarrollo social y económico. Este método observa el patrimonio urbano como un bien social, cultural y económico para el desarrollo de las ciudades (Unesco, 2013).

El enfoque del Paisaje Urbano Histórico se desarrolla en Europa y se proyecta universalmente a través de las conferencias de la Unesco; este desarrollo es debido quizá a que una gran mayoría de los sitios urbanos declarados como Patrimonio Mundial se encuentran en dicho continente.

Es más fácil satisfacer múltiples usos cuando son compatibles o complementarios (por ejemplo la protección de la calidad del agua, la protección de espacios ambientalmente frágiles y la recreación pa- 
siva), pues pueden coexistir sobre la misma superficie. Kato y Ahern (2009) sostienen que incluso los usos en competencia o en conflicto pueden coexistir a partir de estrategias y esquemas de gestión inteligentes, como la secuencia de ocupación temporal utilizada para gestionar los recursos naturales para múltiples usos y objetivos.

Los conceptos de patrimonio cultural y paisaje tienen en común no solo el origen moderno de su significado actual, sino también la visión de conjunto que pretenden ofrecer, la cual ha venido evolucionando a lo largo de caminos paralelos. La tesis de estudio sugiere que ambos conceptos son complementarios y de este modo una colaboración metodológica entre ambas perspectivas es posible. La visión y enfoque comunes se pueden complementar en la búsqueda del desarrollo urbano utilizando la herramienta del planeamiento.

El enfoque de paisaje se vuelve participativo en el sentido que no solo se promueve la información y consulta, sino que se busca empoderar a las comunidades involucradas, incluyéndolas en la toma de

\section{Bibliografía}

decisiones y en la elaboración de los instrumentos de gestión.

Antrop, M. (2000). Background concepts for integrated landscape analysis. Agriculture, Ecosystems and Management, 77 , pp. $17-28$.

Antrop, M. (2004). Why landscapes of the past are important for the future. Landscape and Urban Planning, 1-2, pp. $21-34$.

Antrop, M. (2005). From holistic landscape synthesis to transdisciplinary landscape management. En B. Tress, G. Tress, G. Fry \& P. Opdam. (Eds). From landscape research to landscape planning: aspects for integration, education and application. Heidelberg: Springer.

Antrop, M. (2008). Chapter 4: Landscapes at risk: about change in the European landscapes. En Dostál, P. (Ed). Evolution of Geographical Systems and Risk Processes in the Global Context. Praga, Republica Checa: P3K y Charles University.

Bandarin F. \& Van der Oers, R. (2011). The historic urban landscape, managing heritage in an urban century. Oxford:Wiley-Blackwell.

Fairclough G. (2004). History and time: managing landscape and perceptions. Ponencia presentada en la conferencia
From Knowledge of Landscapes to Landscaping Action. Burdeos, Francia.

Icomos. (2004). History of the Venice Charter. Última revisión: 6 julio 2014.

www.icomos.org/venicecharter2004/history.pdf

Jokilehto, J. (1986). A history of architectural conservation. (PhD Thesis). Institute of Advanced Architectural Studies, University of York, England.

Kato, S. \& Ahern, J. (2008). Learning by doing: adaptive planning as a strategy to address uncertainty in planning. Journal of Environmental Planning and Management, 51, (4), pp.543 - 559.

Kato S. \& Ahern J. (2009). Multifunctional Landscapes a Basis for Sustainable Landscape Development. Landscape Research Journal, 72, (5), pp.799 - 804.

Kwa, C. (2005). Alexander Von Humboltd's invention of the natural landscape. The European Legacy, 10, (2), pp. $149-162$

Construcción de hotel en Cusco genera conflicto entre autoridades de Cultura. (2014, 21 mayo 2014). La República. Recuperado de: http://www.larepublica. pe/21-05-2014/construccion-de-hotel-en-cusco-genera-conflicto-entre-autoridades-de-cultura

Sonkoly, G. (2010). Historic urban landscape: a conceptual analysis. Documento de Trabajo. Última revisión: 11 julio 2014. http://www.academia.edu/1184490/Historic_Urban_Landscape_-_A_Conceptual_Analysis

The Getty Conservation Institute. (2014). Cultural Heritage Policy Documents. General Conclusions of the Athens Conference (1931). Recuperado el 6 Julio 2014 de:

http://www.getty.edu/conservation/publications_resources/ research resources/charters/charter02.html

Tress, B.; Tress, G. \& Fry, G. (2005). Defining concepts and the process of knowledge production in integrative research. En B. Tress, G. Tress, G. Fry \& P. Opdam. (Eds). From landscape research to landscape planning: aspects for integration, education and application. Heidelberg: Springer.

Trott, C. (2008). Managing change in the urban World Heritage context: Probable impacts on site management of the new historic urban landscape category. Case study: Quedlinburg. (Tesis de Maestría). Universidad Tecnológica de Brandenburgo, sede Cottbus, Alemania.

Unesco. (2011). Recommendation on the historic urban landscape. Recuperado el 11 julio 2014 de: http://whc. unesco.org/en/activities/638

Unesco. (2013). Nueva vida para las ciudades históricas: una explicación del paisaje histórico urbano. París, Francia: (s.n.)

United Nations. (1995). Second United Nations Conference on Human Settlements (Hábitat II). Estambul: autor.

United Nations, Population Division 2011. World Urbanization Prospects, the 2011 Revision. Recuperado el 28 de junio 2014 de: http://esa.un.org/unup/Country-Profiles/ country-profiles_1.htm 